\title{
3-Phosphoglycerate dehydrogenase expression is regulated by HOXA10 in murine endometrium and human endometrial cells
}

\author{
Hongling Du ${ }^{1}$, Danielle Vitiello ${ }^{1}$, Jennifer L Sarno ${ }^{1}$ and Hugh S Taylor ${ }^{1,2}$ \\ ${ }^{1}$ Department of Obstetrics, Gynecology and Reproductive Sciences and ${ }^{2}$ Molecular, Cellular and Developmental \\ Biology, Yale University, PO Box 208063, 333 Cedar Street, New Haven, Connecticut 06520, USA
}

Correspondence should be addressed to H S Taylor at Department of Obstetrics, Gynecology and Reproductive Sciences, Yale University; Email: hugh.taylor@yale.edu

\begin{abstract}
3-Phosphoglycerate dehydrogenase (PHGDH, 3-PGDH) is an enzyme necessary for de novo L-serine biosynthesis. HOXA10 expression is required for endometrial receptivity; however, few target genes of HOXA10 regulation are known. Using a microarray we identified Phgdh as a target of HOXA10 regulation in murine endometrium and confirmed this regulatory relationship in human endometrial cells. PHGDH was downregulated 2.0-fold by HOXA10 and upregulated 4.4-fold by HOXA10 antisense in vivo. In human endometrial cells, real-time PCR results show that pcDNA3.1/HOXA10 transfection decreased PHGDH mRNA expression to $40 \%$ of pretreatment level $(P<0.05)$, while $P H G D H$ mRNA expression was increased 2.1 -fold $(P<0.05)$ by HOXA10 siRNA. Western blot results confirmed the regulatory relationship in both primary human endometrial stromal and epithelial cells, as well as in human endometrial stromal cells and Ishikawa cells. In human cycling endometrial tissue, immunohistochemical results showed that PHGDH expression is relatively high in the proliferative phase in glandular cells and lower in the secretory phase. Here we report novel expression and regulation of PHGDH in murine and human endometrium. PHGDH is expressed in both endometrial epithelial and stromal cells. HOXA10 represses endometrial PHGDH expression. PHGDH is necessary for serine biosynthesis, which serves as a substrate for protein synthesis. One mechanism by which HOXA10 regulates cellular differentiation may involve limiting protein synthesis in the secretary phase.

Reproduction (2010) 139 237-245
\end{abstract}

\section{Introduction}

The limiting step of implantation is the ability of the blastocyst to implant into a receptive endometrium. Transient, predictable molecular events within the endometrial glands and stroma render a normally hostile environment receptive to implantation. Thus, the cascade of signaling events that occur in both fetal and maternal tissues at the time of implantation establishes an appropriate milieu critical to the development and survival of the fetus. Defects in formation of this network and the inability to sustain this crosstalk are believed to result in various implantation-associated issues that may manifest as implantation failures or as pregnancy complications throughout gestation (Aplin 2000, Red-Horse et al. 2004).

The homeobox (Hox) genes encode transcription factors that guide embryologic development as well as regulate differential gene expression within the endometrium with each menstrual cycle (Taylor et al. 1997). In particular, a member of this family, HOXA10, encodes a transcription factor required for both endometrial receptivity and blastocyst implantation. The HOXA10 gene product is expressed by endometrial glands and stroma throughout the menstrual cycle (Taylor et al. 1998, 1999) with peak expression coincident with the implantation window of the midsecretory phase (Taylor et al. 1997, Sarno et al. 2005).

Selective alteration of endometrial Hoxa10 expression in mice through the use of liposome-mediated gene transfection dramatically alters implantation (Bagot et al. 2000). While similar studies have not yet been performed in higher animal models or humans, transfection of a human endometrial adenocarcinoma cell line (Ishikawa cells) with a HOXA10 antisense oligodeoxyribonucleotide also resulted in decreased HOXA10 expression (Bagot et al. 2000). Furthermore, efficient transfection and expression of an Escherichia coli lacZ reporter gene has been accomplished in intact human uteri ex vivo (Daftary \& Taylor 2003, 2004). Thus, differential maternal expression of endometrial HOXA10 is essential for implantation as this transcription factor regulates the molecular switches that promote the appropriate endometrial receptivity.

To identify candidate genes that may be regulated by differential HOXA10 expression, we employed our mouse model that allows for transient expression of plasmid-originated select gene products during the 
peri-implantation period (Bagot et al. 2000). Employing a microarray screen, we selected for candidate gene products that were regulated differentially when HOXA10 was transiently overexpressed in endometrium in comparison to vehicle only control tissues (Vitiello et al. 2008). Many of these genes demonstrating altered expression represent commonly occurring gene products within the cell rather than unique regulatory products. The cellular ontogenies of differentially expressed genes included cell adhesion molecules, signal transduction factors as well as metabolic regulators.

3-Phosphoglycerate dehydrogenase (PHGDH, 3-PGDH), a key enzyme necessary for de novo L-serine biosynthesis (Ichihara \& Greenberg 1957), demonstrates clear differential expression patterns in the periimplantation mouse uterus. The human PHGDH gene is regulated at the transcriptional level in a tissues and it is dependent on cellular proliferation status (Cho et al. 2000). It is the first committed enzyme for the de novo phosphorylated pathway of serine synthesis catalyzing the transition of 3-phosphoglycerate into 3-phosphohydroxypyruvate using NAD + as a cofactor and resulting in an increase in the serine pool. Serine itself, a nonessential amino acid, plays a central role in cellular proliferation serving as an indispensable precursor for the synthesis of biomolecules, such as proteins, membrane lipids, other amino acids, and nucleotides (Snell \& Weber 1986, Narkewicz et al. 1996, Xue et al. 1999). It is expressed at high levels in diverse tissue including: prostate, testis, ovary, brain, liver, kidney and pancreas (Snell 1984). The activity of $\mathrm{PHGDH}$ is elevated in some cancers, such as rat hepatomas, rat sarcoma and human colon carcinoma (Snell \& Weber 1986, Snell et al. 1987, 1988).

Hox genes were first recognized as an evolutionarily conserved family of transcription factors critical to the control of early embryonic development. Their differential regulation in human endometrium leads to tissue specialization (Taylor et al. 1998). Estrogen and progesterone regulate HOXA10 expression in both the embryonic and the adult reproductive tracts (Taylor et al. 1998, Block et al. 2000). HOXA10 expression is required for endometrial receptivity, however few target genes of HOXA10 regulation are known. Using microarray we identified Phgdh as a target of HOXA10 in murine endometrium and confirmed this regulatory relationship in human endometrial cells.

\section{Results}

\section{Phgdh is a target of HOXA10 regulation in murine} endometrium

To identify putative gene regulated by HOXA10, we performed an initial screen of these targets employing a microarray. Thirty-nine thousand unique mouse transcripts were probed for differences in expression. The Phgdh gene product was noted to be reciprocally regulated according to HOXA10 expression. When HOXA10 was overexpressed, the microarray results demonstrated an almost 2.0-fold decrease in Phgdh mRNA expression. Conversely, transfection of an enzyme resistant Hoxa10 antisense oligonucleotide demonstrated a 4.4-fold increase in Phgdh mRNA expression (Fig. 1).

\section{PHGDH protein expression in human cycling endometrial tissue}

To determine the expression pattern of PHGDH through the menstrual cycle, we used immunohistochemical analysis on human endometrial samples which were collected from subjects in secretory phase and proliferative phases of the menstrual cycle. PHGDH protein was found in the cytoplasm of both stromal and glandular cells in human endometrium. Immunohistochemical results show PHGDH expression is relatively high in the proliferative phase $(\mathrm{P})$ and lower in the secretory phase (S) in glandular epithelial cells of human cycling endometrium. Immunohistochemical results also show that PHGDH expression is relatively lower in endometrial stromal cells than in glandular epithelial cells in the proliferative phase. PHGDH expression is also lower in the secretory phase than the proliferative phase in stromal cells of human cycling endometrium (Fig. 2 and Table 1).

\section{HOXA10 regulated PHGDH mRNA expression in human endometrial cell lines and primary human endometrial cells}

To confirm the regulatory relationship identified in the murine microarray, we first used the human endometrial stromal cell line (HESC) and the human endometrial epithelial cell line Ishikawa. We have previously described HOXA10 gene expression in HESC and Ishikawa cells (Taylor et al. 1998). HOXA10 mRNA

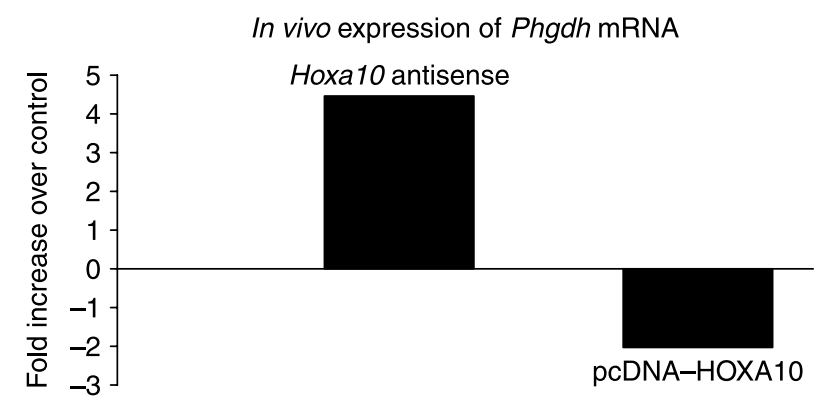

Figure 1 Identification of Phgdh as a target of HOXA10 in murine endometrium as assessed by microarray analysis. Murine uterine endometrium was transfected with a Hoxa10 expression vector or enzyme-resistant Hoxa10 antisense oligonucleotides in vivo and then microarray analysis was performed on RNA extracted from those tissues. Phgdh was downregulated by HOXA10 2.0-fold and upregulated by Hoxa10 antisense 4.4-fold in the murine microarray. 

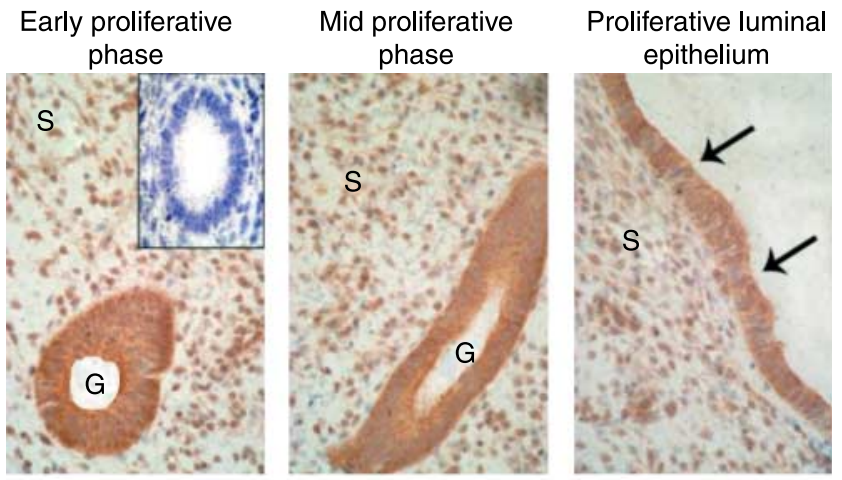

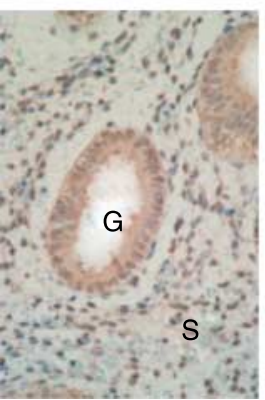

Early secretory phase

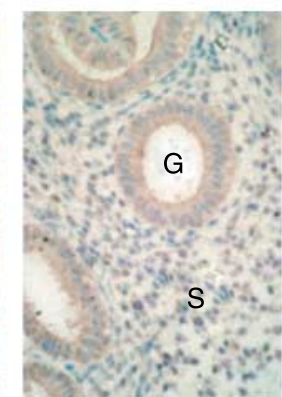

Mid secretory phase

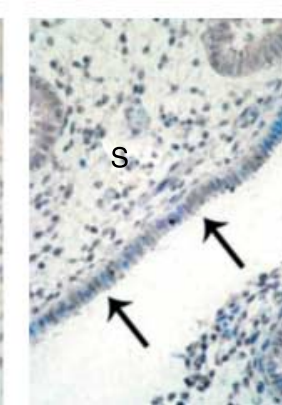

Secretory luminal epithelium
Figure 2 PHGDH protein expression in human cycling endometrium tissue. PHGDH protein was expressed in the cytoplasm of both stromal and glandular cells in human endometrium. Immunohistochemical results show $\mathrm{PHGDH}$ expression is relatively high in the proliferative phase and lower in the secretory phase. The left column shows the first half of each phase, the center column shows the mid portion of each phase (including the window of implantation). The right column shows the luminal epithelial surface at each phase. Arrows point to the luminal epithelial surface ( $\mathrm{S}$, stroma; $\mathrm{G}$, glands). The insert shows a negative control. Photomicrographs taken at $\times 600$ magnification.

was not endogenously expressed in the ovarian cancer cell line A2780, and therefore this cell line was used as a control (Fig. 3A). Quantitative real-time RT-PCR (qRTPCR) results demonstrated that HOXA10 gene expression increased over 1000-fold after transfection with the pcDNA-HOXA10 vector in HESC and Ishikawa cell lines. HOXA10 mRNA expression was decreased $40-50 \%$ of pretreatment level after transfection of HOXA10 siRNA in Ishikawa cell lines, and $60-70 \%$ of pretreatment level after transfection of HOXA10 siRNA in HESC cells (Fig. 4A). As a control A2780 cells were transfected with the HOXA10 expression vector or siRNA (Fig. 3). HOXA10 was not endogenously

Table $1 \mathrm{H}$-score analysis (mean \pm s.E.M).

\begin{tabular}{llll}
\hline & Stroma & Glands & P values \\
\hline $\begin{array}{l}\text { Proliferative phase } \\
(n=10)\end{array}$ & $2.63 \pm 0.253$ & $3.20 \pm 0.228$ & $P<0.05$ \\
$\begin{array}{c}\text { Secretory phase } \\
(n=10)\end{array}$ & $1.78 \pm 0.032$ & $2.165 \pm 0.225$ & $P<0.05$ \\
& $P<0.05$ & $P<0.05$ & \\
\hline
\end{tabular}

expressed by these cells, and could be induced by transfection with pcDNA3.1/HOXA10. PHGDH expression was not changed by transfection with either the HOXA10 expression vector or HOXA10 siRNA in the A2780 cell line. However, pcDNA3.1/HOXA10 transfection did result in decreased $P H G D H$ mRNA expression to $40 \%$ of pretreatment level $(P<0.05)$ in HESC cells, a small increase in PHGDH mRNA expression was seen after HOXA10 siRNA transfection in these cells (Fig. 4B). PHGDH mRNA expression was increased 2.1 -fold $(P<0.05)$ by HOXA10 siRNA transfection in Ishikawa cells (Fig. 4B). There was no significant change in $P H G D H$ mRNA expression after pcDNA3.1/HOXA10 transfection in Ishikawa cells (Fig. 4B).

We also used the primary human endometrial stromal and epithelial cells to confirm the regulatory relationship identified in the cell lines. qRT-PCR results demonstrated that HOXA10 gene expression increased over 1000-fold after transfection with the pcDNA-HOXA10 vector in primary human endometrial cells. HOXA10 mRNA expression was decreased around $70 \%$ of pretreatment level after transfection of HOXA10 siRNA in both primary human endometrial stromal and epithelial cells (Fig. 5A). pcDNA3.1/HOXA10 transfection result in decreased $P H G D H$ mRNA expression to $70 \%$ of pretreatment level $(P<0.05)$ in primary HESC (Fig. 5B). $P H G D H$ mRNA expression was increased 1.7-fold $(P<0.05)$ by HOXA10 siRNA transfection in primary human endometrial epithelial cells (Fig. 5B).

\section{HOXA10 regulated PHGDH protein expression in human endometrial cell lines and primary human endometrial cells}

Western blot results demonstrated that HOXA10 gene expression increased after transfection of the pcDNAHOXA10 vector and decreased after transfection with HOXA10 siRNA in the HESC, Ishikawa cells and primary human endometrial cells (Fig. 6a). Figure 6b shows western blot results after pcDNA/HOXA10 transfection of HESC and Ishikawa cells; PHGDH protein expression was decreased in these cells. Densitometry revealed that HOXA10 transfection significantly decreased PHGDH protein expression by $62 \pm 5.2$ and $30 \pm 3.2 \%$ in HESC and Ishikawa cells, respectively. After siRNA transfection, $\mathrm{PHGDH}$ protein expression was increased in these cells. siRNA treatment increased $\mathrm{PHGDH}$ protein by 70 \pm 12.4 and $45 \pm 5.6 \%$, respectively, in these cells. Similarly, PHGDH protein expression was increased by HOXA10 siRNA transfection in primary human endometrial cells. Densitometry revealed a $25 \pm 2.6$ and 40 $\pm 1.7 \%$ increase in PHGDH expression in primary stromal and epithelial cells respectively. There was a similar decrease in $\mathrm{PHGDH}$ protein expression in primary human endometrial cells after pcDNA3.1/HOXA10 transfection, indicating submaximal repression at 

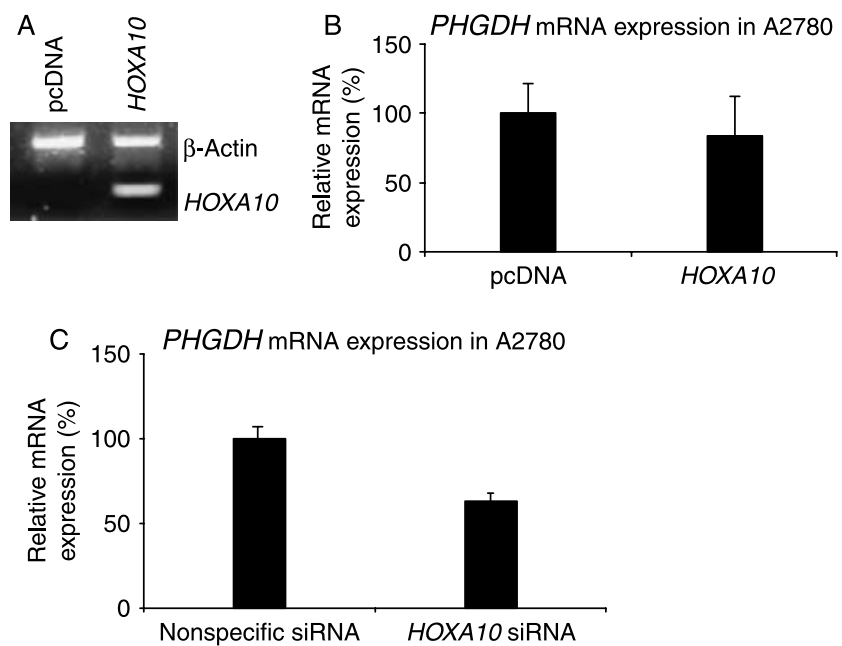

Figure 3 HOXA 10 and $P H G D H$ mRNA expression in the control A2780 ovarian cancer cell line. (A) RT-PCR results show absent HOXA10 mRNA expression in A2780 cells after transfection with empty pcDNA vector. HOXA10 mRNA expression was seen only after transfection with the HOXA10 vector. (B) PHGDH mRNA expression was not regulated in the $\mathrm{A} 2780$ cell line after transfection with the pcDNA-HOXA10 vector. (C) PHGDH mRNA expression was not regulated in the $\mathrm{A} 2780$ cell line after transfection with the HOXA10 siRNA. Shown are mean \pm s.D.

endogenous levels of HOXA10 expression. Densitometry revealed a $33 \pm 6.1$ and $23 \pm 3.1 \%$ decrease in PHGDH expression in primary stromal and epithelial cells respectively. Taken together, these data confirmed the regulatory relationship determined in both HESC and Ishikawa cells in mRNA expression. As a control, A2780 cells were transfected with the HOXA10 expression vector. HOXA10 protein could be induced by transfection with pcDNA3.1/HOXA10. PHGDH protein expression was not changed by transfection with either the HOXA10 expression vector or HOXA10 siRNA in the A2780 cell line.

\section{Discussion}

Uterine endometrium undergoes dramatic proliferation and growth, regenerating completely each menstrual/ estrus cycle. The molecular signaling pathways that regulate this growth and subsequent differentiation are still incompletely characterized. Few genes are known to be essential for this process. It is intuitive that genes regulating metabolism and protein synthesis would be required for these functions; however, few have been identified and the regulation of their expression has not been described. The PHGDH gene encodes PHGDH, which catalyzes the first step in the phosphorylated pathway of serine biosynthesis.

In the adult, the expression of this gene has been associated with cellular proliferative status. In monocytic cells, for example, experimentally-induced cell differentiation results in growth arrest and abrupt downregulation of PHGDH (Cho et al. 2000). Regulation of enzymes that are necessary for protein synthesis is linked to proliferative potential. Our data suggest that a similar mechanism exists in the endometrium, where $\mathrm{PHGDH}$ is downregulated in the secretory phase. It is necessary for a cell to have the ability to regulate enzymes essential for distinct cellular functions
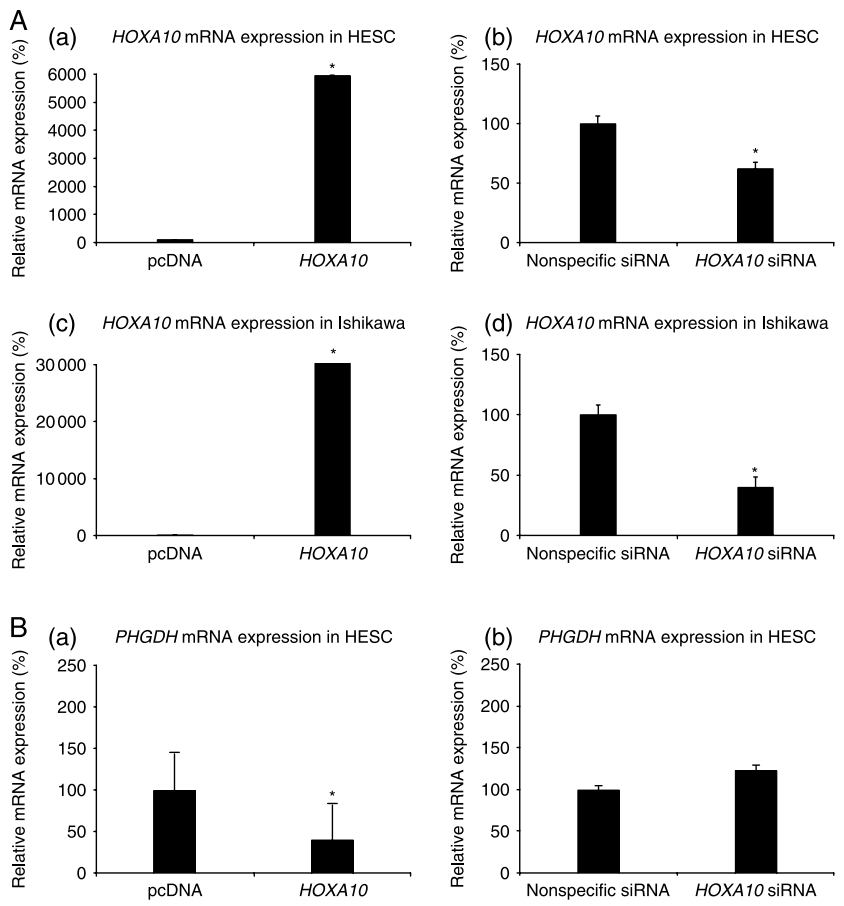

(c) PHGDH mRNA expression in Ishikawa
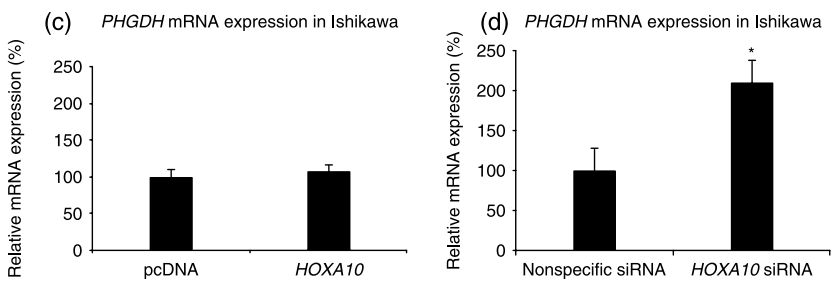

Figure 4 (A) HOXA10 mRNA expression in human endometrial cell lines. (a) Real-time PCR results show that pcDNA3.1/HOXA10 transfection increased HOXA10 mRNA expression over 1000-fold $(P<0.05)$ in HESC cells. (b) HOXA10 mRNA expression decreased to $60 \%(P<0.05)$ of pretreatment level after transfection of HOXA10 siRNA in HESC cells. (c) Similarly, real-time PCR results show that HOXA10 mRNA expression was increased over 1000 -fold $(P<0.05)$ by pcDNA3.1/HOXA10 transfection in Ishikawa cells. (d) HOXA10 mRNA expression decreased to $\sim 40 \%(P<0.05)$ of pretreatment level after transfection with HOXA10 siRNA in Ishikawa cells. Shown are mean \pm s.D. ${ }^{*}$ Indicates $P<0.05$. (B) HOXA10 regulated $P H G D H$ mRNA expression in human endometrial cell lines. (a) Real-time PCR results show that pcDNA3.1/HOXA10 transfection decreased $P H G D H$ mRNA expression to $40 \%$ of pretreatment level $(P<0.05)$ in HESC cells. (b) PHGDH mRNA expression was not significantly increased after transfection of HOXA10 siRNA in HESC cells. (c) PHGDH mRNA expression was not significantly changed after transfection with pcDNA3.1/HOXA10 in Ishikawa cells. (d) Real-time PCR results show that $P H G D H$ mRNA expression was increased 2.1 -fold $(P<0.05)$ by HOXA10 siRNA transfection in Ishikawa cells. Shown are mean \pm s.D. *Indicates $P<0.05$. 

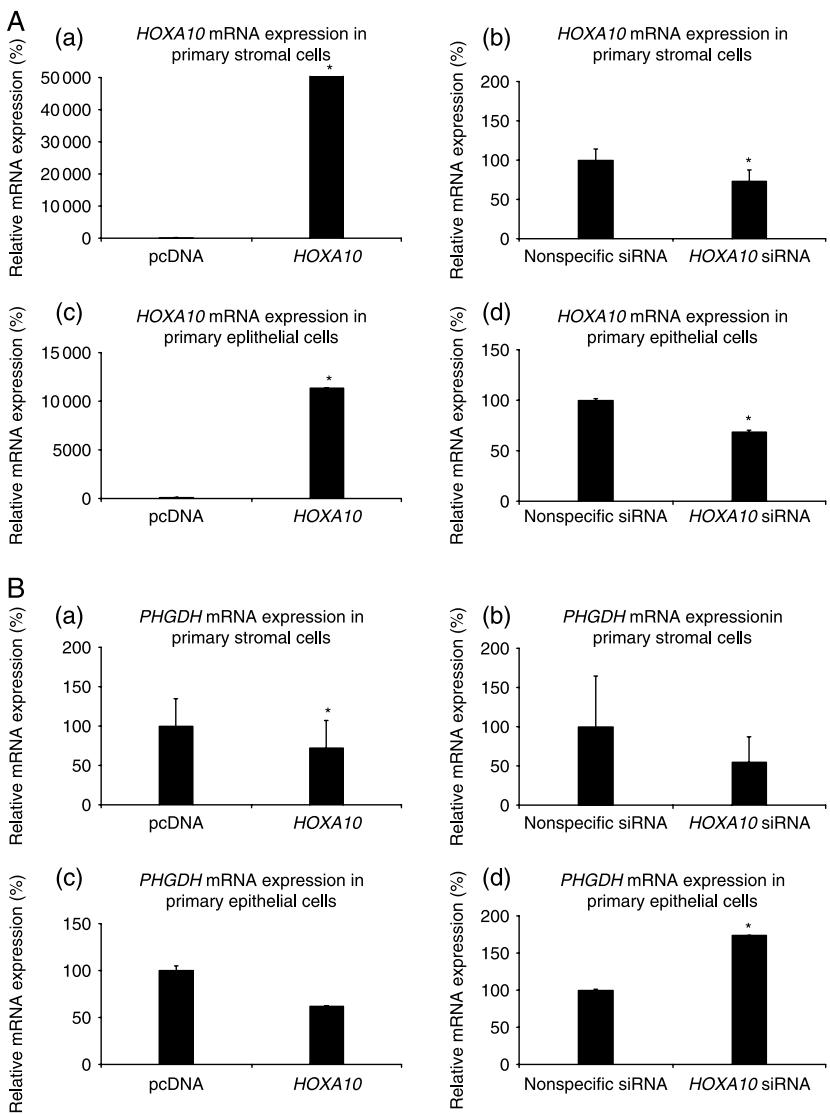

Figure 5 (A) HOXA10 mRNA expression in primary human endometrial cells. (a) Real-time PCR results show that pcDNA3.1/HOXA10 transfection increased HOXA10 mRNA expression over 1000-fold $(P<0.05)$ in primary human endometrial cells. (b) HOXA10 mRNA expression decreased around $70 \%(P<0.05)$ of pretreatment level after transfection with HOXA10 siRNA in primary human endometrial cells. (c) Similarly, real-time PCR results show that HOXA10 mRNA expression increased over 1000 -fold $(P<0.05)$ after pcDNA3.1/ HOXA10 transfection in primary endometrial epithelial cells. (d) HOXA10 mRNA expression decreased to $70 \%(P<0.05)$ of pretreatment level after HOXA10 siRNA transfection in primary human endometrial epithelial cells. Shown are mean \pm s.D. ${ }^{*}$ Indicates $P<0.05$. (B) HOXA10 regulated PHGDH mRNA expression in primary human endometrial cells. (a) Real-time PCR results show that pcDNA3.1/ HOXA10 transfection decreased $P H G D H$ mRNA expression around $70 \%$ of pretreatment level $(P<0.05)$ in primary human endometrial cells. (b) $P H G D H$ mRNA expression showed no significant increase after transfection of HOXA10 siRNA in primary human endometrial cells. (c) PHGDH mRNA expression was not significantly changed after transfection with pcDNA3.1/HOXA10 in primary human endometrial cells. (d) Real-time PCR results show that PHGDH mRNA expression was increased 1.7 -fold $(P<0.05)$ by HOXA10 siRNA transfection in Ishikawa cells. Shown are mean \pm s.D. ${ }^{*}$ Indicates $P<0.05$.

throughout the course of the reproductive cycle; however, the molecular mechanisms responsible for this regulation are largely unknown.

HOXA10 is a transcription factor necessary for endometrial receptivity demonstrating heightened endometrial expression during the implantation window. Previously, we have reported that HOXA10 regulates the expression of other transcription factors and molecules that are important for embryo attachment at the epithelial cell surface (Daftary et al. 2002, Troy et al. 2003). Targeted disruption of Hoxa10 in mice results in loss of endometrial receptivity (Satokata et al. 1995) while diminished expression in mice or humans similarly leads diminished embryo implantation (Bagot et al. 2000, Daftary et al. 2002, Cermik et al. 2003).

Here we demonstrate that HOXA10 also has a regulatory effect on genes necessary for regulation of protein synthesis. We speculate that HOXA10 is involved in modulating epithelial cell metabolism. When proliferation and demand for protein synthesis is at its peak, there is minimal glandular HOXA10 geneproduct expression; and consequently, there is no repression of enzymes (such as $\mathrm{PHGDH}$ ) needed for protein synthesis, thus meeting the increasing metabolic demands. However, in the secretory phase, the glands' cellular contents have been expelled into the uterine lumen, glandular proliferation ceases, and the demand for new protein synthesis is minimal. As the concentrations of HOXA10 gene product increases, the production of PHGDH decreases.

Not surprisingly, the in vitro results reflect the underlying physiology where HOXA10 represses PHGDH expression. In Ishikawa cells, HOXA10 siRNA treatment leads to derepression of PHGDH expression as predicted. Treatment with excess HOXA10 had less effect on expression of PHGDH, likely reflecting some repression by the endogenous HOXA10 expressed by Ishikawa cells. The endogenous level of HOXA10 in these cells is higher than that seen in the proliferative phase endometrial epithelial cells. Conversely, in stromal cells there is a low level of PHGDH expression in both proliferative and secretory phases.

Employing HESC cells, we demonstrate that siRNA treatment had minimal effect; these data imply that co-repressors may govern PHGDH expression even in the absence of HOXA10. Additionally, excess HOXA10 expression leads to further suppression. Such transcriptional regulation would explain the persistently low expression in stromal cells. These findings support an additional repressive mechanism whereby PHGDH expression is limited significantly during the implantation window and that the degree of enzyme synthesis repression varies within this timeframe. It appears that overexpression of HOXA10 gene product affords additional PHGDH repression, leading to the near absence of expression seen in the secretory phase.

In vivo we demonstrate that in human cycling endometrium tissue, PHGDH expression is relative high in the proliferative phase in glandular cells and lower in the secretory phase. HOXA10 represses endometrial PHGDH expression. PHGDH expression and therefore function were inhibited by HOXA10 in the secretory phase in human cycling endometrium, a phase in which endometrial cells growth is suppressed. In the 
proliferative phase, HOXA10 expression decreases and PHGDH expression was derepressed facilitating endometrial cell growth. In vitro, we show that PHGDH expression is similarly regulated by HOXA10 expression in isolated primary human endometrial cells. PHGDH was downregulated by HOXA10 overexpression in primary HESC, and upregulated by HOXA10 repression in primary human endometrial epithelial cells.

This dynamic regulation of $P H G D H$ gene expression by HOXA10 provides for specific cellular metabolic profiles to meet the changing needs of the local environment. Specifically, heightened levels of the
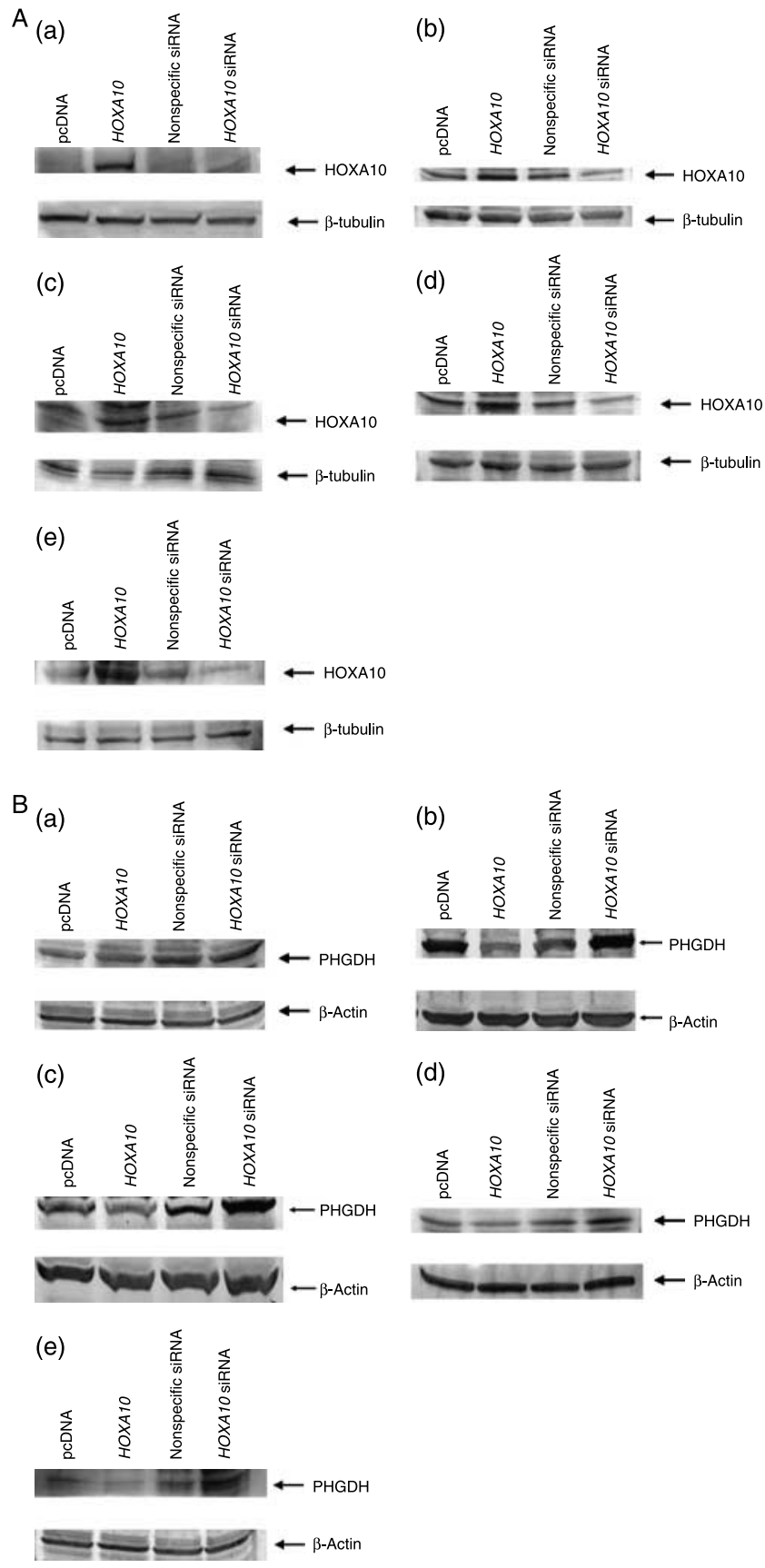

enzyme provide a potential nondietary and nonhepatic source of serine and glycine at a time in the reproductive cycle when protein production is necessary for proliferation and generation of a receptive endometrium. Linking the regulation of endometrial receptivity and cellular metabolic demands through a single transcription factor assures synchrony of these events. Data presented here contribute to defining the molecular signature of uterine receptivity during early mouse implantation. Further characterization of these genes and their role in implantation may assist to elucidate the complex molecular and physiologic events that govern reproductive success.

\section{Materials and Methods \\ Plasmids, oligonucleotides and siRNA}

HOXA10 cDNA was cloned into the EcoRI site of pcDNA3.1 $(+)$ (Invitrogen). PcDNA3.1(+) without the HOXA10 insert was used as a control (Invitrogen). Two 30-mer phosphothiorate-protected oligodeoxyribonucleotides were synthesized by the W M Keck Oligonucleotide Laboratory at Yale University; one oligonucleotide complementary to the start of translation of Hoxa10/HOXA10 corresponding to nucleotides 44-73 (GenBank accession number L08757): 5'-CTCTCCGAGCATGACATTGTTGTGGGATAA-3' (antisense); the second has the same nucleotide composition but a random sequence and was used as a missense control: 5'-TGCTGCTAGGATCGTTCAAGTGTATCACGA-3'. The efficacy of the HOXA10 expression vector and the HOXA10 antisense molecule have been tested and validated as previously described

Figure 6 (A) HOXA10 protein expression in human cell lines and primary human endometrial cells. (a) Western blot results show that pcDNA/HOXA10 transfection induced HOXA10 protein expression in A2780 cells. (b) HOXA10 protein expression was increased after transfection with HOXA10 and decreased after transfection with HOXA10 siRNA in HESC. (c) Similarly, HOXA10 protein expression was increased after transfection with HOXA10 and decreased after transfection with HOXA10 siRNA in Ishikawa cells. (d) HOXA10 protein expression was increased by pcDNA3.1/HOXA10 transfection and was decreased by HOXA10 siRNA transfection in primary human endometrial cells. (e) HOXA10 protein expression was increased after transfection with pCDNA3.1/HOXA10 and decreased after transfection with HOXA10 siRNA in primary human epithelial cells. (B) HOXA10 regulated $\mathrm{PHGDH}$ protein expression in human cell lines and primary human endometrial cells. (a) Western blot results show that PHGDH protein expression was not changed in A2780 cells after pcDNA3.1/ HOXA10 and HOXA10 siRNA. (b) pcDNA/HOXA10 transfection decreased PHGDH protein expression and HOXA10 siRNA transfection increased PHGDH expression in HESC ells. (c) Western blot results also show that $\mathrm{PHGDH}$ protein expression was increased by HOXA10 siRNA transfection and decreased by HOXA10 expression in Ishikawa cells. (d) Similarly, western blot results show that PHGDH protein expression was increased by HOXA10 siRNA transfection and decreased by HOXA10 expression in primary human endometrial cells. (E) PHGDH protein expression was increased by HOXA10 siRNA transfection and decreased by HOXA10 expression in primary human epithelial cells. 
(Bagot et al. 2001). SiGenome duplex HOXA10 siRNA (cat\# D-006336-01) and control nontargeting siRNA (cat\# D-00121002) were purchased from Dharmacon (Chicago, IL, USA).

\section{In vivo gene transfection and tissue microarray}

Nulliparous reproductive age female and male CD1 mice were obtained from Charles River (Wilmington, MA, USA). Three animals were used for each test condition. Mice were mated and examined every $12 \mathrm{~h}$ until the detection of a vaginal plug. Twenty-four hours after plug detection, a laparotomy was performed. The mice first were anesthetized with $0.1 \mathrm{ml} / 10 \mathrm{~g}$ of a xylazine $(10 \mathrm{mg} / \mathrm{kg}) / \mathrm{ketamine}(100 \mathrm{mg} / \mathrm{kg})$ mixture given by i.p. injection. Each uterine horn was injected with $25 \mu$ of a preincubated DNA/lipofectamine (Bagot et al. 2000, Vitiello et al. 2008). Liposome-mediated gene transfection was used to deliver either pcDNA-HOXA10/pcDNA3.1 $(+)$ vector $(n=3)$ or an enzyme-resistant Hoxa10 antisense or missense oligonucleotide to the murine uterus $(n=3)$. A phosphorthioate-DNAoligonucleotide (CTCTCCGAGCATGACATTGTTGTGGGATAA) complementary to the translational start site of the Hoxa10 transcript (nucleotides 44-73) was used in addition to a missense oligonucleotide of the same nucleotide composition but in scrambled order. The peritoneum was closed separately from the skin incision employing a running 3-0 vicryl stitch. Forty-eight hours post-laparotomy, the uterus was excised and total uterine RNA was isolated using Trizol (Invitrogen) and RNA-easy kit (Qiagen) according to manufacturer's directions. Purified RNA was analyzed using the Affymetrix mouse genome 4302.0 array which probes for 39000 gene products. Gene Spring 6.0 was used to perform a two-sided $t$-test and genes were classified according to functional ontologies. Those candidates demonstrating statisitically significant changes in expression profiles were selected for further assessment. These experiments were conducted in accordance with an approved protocol issued by the Yale Animal Care and Use Committee.

\section{Immunohistochemistry}

Endometrial samples were collected from 20 normally cycling reproductive age women (age 28-40, proliferative phase, $n=10$; secretory phase, $n=10$ ) not using hormonal therapy, throughout the menstrual cycle under a Human Investigation Committee approved protocol. Formalin-fixed paraffinembedded tissues were cut into $5 \mu \mathrm{m}$ thick sections and mounted on coated slides. Slides were de-paraffinized and rehydrated through a series of xylene and ethanol washes, followed by permeabilization in 95\% cold ethanol. After a 5 min rinse in distilled water, an antigen-presenting step was performed by steaming the slides in $0.01 \mathrm{mM}$ sodium citrate buffer for $20 \mathrm{~min}$, followed by removal of the staining jar from the steam chamber and cooling for $20 \mathrm{~min}$. Slides were rinsed for $5 \mathrm{~min}$ in PBS with $0.1 \%$ Tween20 (PBST20), and sections were circumscribed with a hydrophobic pen. Endogenous peroxidase was quenched with $3 \%$ hydrogen peroxide for 5 min followed by a 5 min PBST wash. Nonspecific binding was blocked with $1.5 \%$ normal horse serum in PBST for $1 \mathrm{~h}$ at room temperature. Slides were incubated in the primary antibody, goat polyclonal HOXA10 antibody at a dilution of
1:200, or polyclonal PHGDH antibody at a dilution of 1:150 overnight at $4{ }^{\circ} \mathrm{C}$. All primary antibodies were purchased from Santa Cruz Biotechnology (Santa Cruz, CA, USA): HOXA10 (sc-17159), PHGDH (sc-16222). Normal goat IgG (Santa Cruz Biotechnology) was used as a negative control. Biotinylated secondary antibodies were purchased from Vector Laboratories (Vector; Burlingame, CA, USA). Horse anti-goat secondary antibody $(3.5 \mu \mathrm{g} / \mathrm{ml})$ for HOXA10 and PHGDH was applied for $1 \mathrm{~h}$ at $4{ }^{\circ} \mathrm{C}$. Slides were washed in $1 \times \mathrm{PBST}$, incubated in ABC Elite (Vector) for $15 \mathrm{~min}$ at room temperature, washed in $1 \times$ PBST, and incubated for $5 \mathrm{~min}$ in diaminobenzidine (DAB; $400 \mathrm{mg} / \mathrm{ml}$; Vector). A $15 \mathrm{~s}$ exposure to hematoxylin was used as a counterstain. For each individual primary antibody, all slides were processed simultaneously. Slides were rehydrated through $3 \mathrm{~min}$ ethanol and xylene washes and mounted with Permount. Expression and localization differences were evaluated and scored on an Olympus BX 40 light microscope by two different observers blinded to the tissue origin using the H-SCORE system (Lessey et al. 1994, Sharpe-Timms et al. 2000). The H-SCORE, representing levels of staining intensity and distribution, is calculated using the following equation: $H$-SCORE $=\Sigma P i(I+1)$, where $I$ is the intensity of staining with a value of 1, 2 or 3 (weak, moderate or strong respectively), and $\mathrm{Pi}$ is the percentage of stained cells for each intensity, varying from 0 to $100 \%$. $H$-score data were analyzed by Mann-Whitney rank sum test.

\section{Cell culture}

The HESC were a generous gift of Dr Charles J Lockwood (Yale University; Krikun et al. 2004); the human endometrial adenocarcinoma cell line (Ishikawa) and human ovarian cancer cell line (A2780) were generous gifts of Dr Richard Hochberg and Dr Gil Mor respectively (Yale University). Primary HESC and primary human endometrial epithelial cells were isolated from human endometrial tissues (Zhang et al. 1995). Previously we have demonstrated that these cells consist of $97 \%$ epithelial cells using Factor VIII, cytokeratin, 3C10, and vimentin as markers of endothelial cells, epithelial cells, macrophages and stromal cells, respectively (Taylor et al. 1998). HESC and primary HESC were maintained in a phenol-red-free DMEM, Ham's F-12 (Sigma), supplemented with 10\% charcoal-stripped calf serum, $1 \%$ penicillin/streptomycin, and 1\% sodium pyruvate. Ishikawa and primary human endometrial epithelial cells were maintained in MEM (Sigma), supplemented with $10 \%$ charcoal-stripped calf serum, $1 \%$ penicillin/streptomycin and $1 \%$ sodium pyruvate. Passage 2-4 of primary human endometrial stromal and epithelial cells was used. A2780 cells were cultured in RPMI 1640 (Invitrogen), supplemented with $10 \%$ charcoal-stripped calf serum and $1 \%$ penicillin/streptomycin, $1 \times$ nonessential amino acid and $1 \%$ sodium pyruvate.

\section{Transient gene transfection in human cell lines}

HESC and primary HESC, grown to $60-70 \%$ confluence, were transfected using TransIT-LT1 Mirus (Madison, WI, USA) with either pcDNA3.1 $(+) / \mathrm{HOXA10}(0.4 \mu \mathrm{g}$ for 6 -well plate, $12 \mu \mathrm{g}$ for $10 \mathrm{~cm}$ dish) or HOXA10 siRNA (20 $\mu \mathrm{M}$ for 6-well plate, $60 \mu \mathrm{M}$ for $10 \mathrm{~cm}$ dish), using empty pcDNA3.1(+) 
or nonspecific siRNA as respective control. Ishikawa cells, A2780 cells and primary human endometrial epithelial cells, grown to $45-55 \%$ confluence, were transfected using lipofectamine 2000 (Invitrogen) with either pcDNA3.1(+)/HOXA10 $(0.4 \mu \mathrm{g}$ for 6-well plate, $12 \mu \mathrm{g}$ for $10 \mathrm{~cm}$ dish) or HOXA10 siRNA (20 $\mu \mathrm{M}$ for 6 -well plate, $60 \mu \mathrm{M}$ for $10 \mathrm{~cm}$ dish), using empty pcDNA3.1(+) or nonspecific siRNA as respective control. After $4 \mathrm{~h}$, the media were changed and cells were incubated for an additional $20 \mathrm{~h}$ in OPTI-MEM. OPTI-MEM I Reduced Serum Medium (Invitrogen), without serum or antibiotics, was used during transfection. Forty-eight hours post-transfection, total RNA and protein were isolated. All transfections were performed in triplicate.

\section{Real-time PCR}

qRT-PCR was performed using iScript cDNA Synthesis Kit and iQ SYBR Green Supermix (Bio-Rad). RNA was reversetranscribed for $30 \mathrm{~min}$ at $42{ }^{\circ} \mathrm{C}$. PCR for HOXA10 was performed for 45 cycles at $95{ }^{\circ} \mathrm{C}$ for $15 \mathrm{~s} ; 61{ }^{\circ} \mathrm{C}$ for $20 \mathrm{~s} ; 72{ }^{\circ} \mathrm{C}$ for $25 \mathrm{~s}$. PCR for PHGDH was performed for 45 cycles of $95^{\circ} \mathrm{C}$ for $15 \mathrm{~s} ; 60^{\circ} \mathrm{C}$ for $20 \mathrm{~s} ; 72{ }^{\circ} \mathrm{C}$ for $25 \mathrm{~s}$. PCR for control $\beta$-actin was performed for 45 cycles of $95^{\circ} \mathrm{C}$ for $15 \mathrm{~s} ; 60{ }^{\circ} \mathrm{C}$ for $20 \mathrm{~s}$; $72{ }^{\circ} \mathrm{C}$ for $25 \mathrm{~s}$. HOXA10 and $\beta$-actin primers were used as previously described (Du et al. 2005). PHGDH primers were 5'-AACTTCTTCCGСТCССАTTT-3' (forward) and 5'-GTCATCAACGCAGCTGAGAA- $3^{\prime}$ (reverse). Expression was adjusted to the expression of $\beta$-actin from the same sample. Melting curve analysis was conducted to determine the specificity of the amplified products and to ensure the absence of primer-dimer formation. All products obtained yielded the predicted melting temperature. Analysis of relative gene expression data used $2^{-\Delta \Delta C_{\mathrm{t}}}$ method (Livak \& Schmittgen 2001). The $C_{\mathrm{t}}$ values were converted to the term $2^{-C_{\mathrm{t}}}$. Group means were evaluated by $t$-test $(n \geq 3)$. Differences of $P<0.05$ were considered significant.

\section{Western blot}

Whole cell protein was extracted from A2780 cells, HESC, Ishikawa cells as well as primary human endometrial stromal and epithelial cells using Nuclear Extract Kit (Activemotif, Carlsbad, CA, USA) according to manufacturer's protocol. Equal amounts of protein $(60 \mu \mathrm{g}$ for both HOXA10 and $\mathrm{PHGDH}$ ) were electrophoresed through 4-15\% polyacrylamide gels (Bio-Rad) at $160 \mathrm{~V}$ for $70 \mathrm{~min}$ and transferred onto Immun-Blot polyvindylidene difluoride membranes (Bio-Rad) in transfer buffer (25 mM Tris, $192 \mathrm{mM}$ glycine, 20\% methanol) at $100 \mathrm{~V}$ for $1 \mathrm{~h}$. After incubation in blocking buffer $(1 \times \mathrm{PBS}$, $0.2 \%$ Tween $20,5 \%$ milk), the membrane was incubated individually with either goat polyclonal HOXA10 antibody (sc-17159) (Santa Cruz Biotechnology) dilution 1:200, or polyclonal PHGDH antibody (sc-16222) (Santa Cruz Biotechnology) dilution 1:200 overnight at $4{ }^{\circ} \mathrm{C}$. Alternatively the membranes were incubated with either goat polyclonal actin antibody (sc-1615) (Santa Cruz Biotechnology) dilution 1:1000, mouse $\beta$-tubulin MAB (sc-8035) (Santa Cruz Biotechnology) at a dilution of 1:1000, at room temperature for $1 \mathrm{~h}$. After washing, the membranes were incubated for $1 \mathrm{~h}$ with biotinylated horse anti-goat secondary antibody or goat anti-mouse secondary antibody (Vector) diluted $(3.5 \mu \mathrm{g} / \mathrm{ml})$ in the blocking buffer. The membranes were incubated in $A B C$ Elite (Vector), and then stained by DAB (Vector). Densitometry was performed using Image J version 1.43a ( $\mathrm{NIH}$, Bethesda, $M D, U S A)$ and normalized to actin expression. Group means were evaluated by $t$-test $(n=3)$. Differences of $P<0.05$ were considered significant.

\section{Declaration of interest}

The authors declare that there is no conflict of interest that could be perceived as prejudicing the impartiality of the research reported.

\section{Funding}

This study was supported by National Institutes of Health Grants HD036887 and HD052668.

\section{References}

Aplin JD 2000 The cell biological basis of human implantation. Best Practice \& Research. Clinical Obstetrics \& Gynaecology 14 757-764.

Bagot CN, Troy PJ \& Taylor HS 2000 Alteration of maternal Hoxa10 expression by in vivo gene transfection affects implantation. Gene Therapy 7 1378-1384.

Bagot CN, Kliman HJ \& Taylor HS 2001 Maternal Hoxa10 is required for pinopod formation in the development of mouse uterine receptivity to embryo implantation. Developmental Dynamics 222 538-544.

Block K, Kardana A, Igarashi P \& Taylor HS 2000 In utero diethylstilbestrol (DES) exposure alters Hox gene expression in the developing Mullerian system. FASEB Journal 14 1101-1108.

Cermik D, Selam B \& Taylor HS 2003 Regulation of HOXA-10 expression by testosterone in vitro and in the endometrium of patients with polycystic ovary syndrome. Journal of Clinical Endocrinology and Metabolism 88 238-243.

Cho HM, Jun DY, Bae MA, Ahn JD \& Kim YH 2000 Nucleotide sequence and differential expression of the human 3-phosphoglycerate dehydrogenase gene. Gene 245 193-201.

Daftary GS \& Taylor HS 2003 Reproductive tract gene transfer. Fertility and Sterility 80 475-484.

Daftary GS \& Taylor HS 2004 EMX2 gene expression in the female reproductive tract and aberrant expression in the endometrium of patients with endometriosis. Journal of Clinical Endocrinology and Metabolism 89 2390-2396.

Daftary GS, Troy PJ, Bagot CN, Young SL \& Taylor HS 2002 Direct regulation of $\beta 3$-integrin subunit gene expression by HOXA10 in endometrial cells. Molecular Endocrinology 16 571-579.

Du H, Daftary GS, Lalwani SI \& Taylor HS 2005 Direct regulation of HOXA10 by $1,25-(\mathrm{OH}) 2 \mathrm{D} 3$ in human myelomonocytic cells and human endometrial stromal cells. Molecular Endocrinology 19 2222-2233.

Ichihara A \& Greenberg DM 1957 Further studies on the pathway of serine formation from carbohydrate. Journal of Biological Chemistry 224 331-340.

Krikun G, Mor G, Alvero A, Guller S, Schatz F, Sapi E, Rahman M, Caze R, Qumsiyeh M \& Lockwood CJ 2004 A novel immortalized human endometrial stromal cell line with normal progestational response. Endocrinology 145 2291-2296.

Lessey BA, Castelbaum AJ, Sawin SW, Buck CA, Schinnar R, Bilker W \& Strom BL 1994 Aberrant integrin expression in the endometrium of women with endometriosis. Journal of Clinical Endocrinology and Metabolism 79 643-649.

Livak KJ \& Schmittgen TD 2001 Analysis of relative gene expression data using real-time quantitative PCR and the $2(-$ Delta Delta $C(\mathrm{~T}))$ method. Methods 25 402-408.

Narkewicz MR, Sauls SD, Tjoa SS, Teng C \& Fennessey PV 1996 Evidence for intracellular partitioning of serine and glycine metabolism in Chinese hamster ovary cells. Biochemical Journal 313 991-996. 
Red-Horse K, Zhou Y, Genbacev O, Prakobhphol A, Foulk A, McMaster M \& Fisher SJ 2004 Trophoblast differentiation during embryo implantation and formation of the maternal-fetal interface. Journal of Clinical Investigation 114 744-754.

Sarno JL, Kliman HJ \& Taylor HS 2005 HOXA10, Pbx2, and Meis1 protein expression in the human endometrium: formation of multimeric complexes on HOXA10 target genes. Journal of Clinical Endocrinology and Metabolism 90 522-528.

Satokata I, Benson G \& Maas R 1995 Sexually dimorphic sterility phenotypes in Hoxa10-deficient mice. Nature 374 460-463.

Sharpe-Timms KL, Ricke EA, Piva M \& Horowitz GM 2000 Differential expression and localization of de novo synthesized endometriotic haptoglobin in endometrium and endometriotic lesions. Human Reproduction 15 2180-2185.

Snell K 1984 Enzymes of serine metabolism in normal, developing and neoplastic rat tissues. Advances in Enzyme Regulation 22 325-400.

Snell K \& Weber G 1986 Enzymic imbalance in serine metabolism in rat hepatomas. Biochemical Journal 233 617-620.

Snell K, Natsumeda Y \& Weber G 1987 The modulation of serine metabolism in hepatoma 3924A during different phases of cellular proliferation in culture. Biochemical Journal 245 609-612.

Snell K, Natsumeda Y, Eble JN, Glover JL \& Weber G 1988 Enzymic imbalance in serine metabolism in human colon carcinoma and rat sarcoma. British Journal of Cancer 57 87-90.

Taylor HS, Vanden Heuvel GB \& Igarashi PA 1997 Conserved Hox axis in the mouse and human female reproductive system: late establishment and persistent adult expression of the Hoxa cluster genes. Biology of Reproduction 57 1338-1345.
Taylor HS, Arici A, Olive D \& Igarashi P 1998 HOXA10 is expressed in response to sex steroids at the time of implantation in the human endometrium. Journal of Clinical Investigation 101 1379-1384.

Taylor HS, Igarashi P, Olive D \& Arici A 1999 Sex steroids mediate HOXA11 expression in the human peri-implantation endometrium. Journal of Clinical Endocrinology and Metabolism 84 1129-1135.

Troy PJ, Daftary GS, Bagot CN \& Taylor HS 2003 Transcriptional repression of peri-implantation EMX2 expression in mammalian reproduction by HOXA10. Molecular and Cellular Biology 23 1-13.

Vitiello D, Pinard R \& Taylor HS 2008 Gene expression profiling reveals putative HOXA10 downstream targets in the periimplantation mouse uterus. Reproductive Sciences 15 529-535.

Xue HH, Sakaguchi T, Fujie M, Ogawa H \& Ichiyama A 1999 Flux of the L-serine metabolism in rabbit, human, and dog livers: substantial contributions of both mitochondrial and peroxisomal serine:pyruvate/ alanine:glyoxylate amino-transferase. Journal of Biological Chemistry 274 16028-16033.

Zhang L, Rees MC \& Bicknell R 1995 The isolation and long-term culture of normal human endometrial epithelium and stroma. Expression of mRNAs for angiogenic polypeptides basally and on oestrogen and progesterone challenges. Journal of Cell Science 108 323-331.

Received 4 September 2008

First decision 17 October 2008

Revised manuscript received 17 September 2009

Accepted 24 September 2009 